\title{
Eine Therapieevaluation zur Behandlung von menopausalen Symptomen mit Padma Nerven-Tonikum
}

\author{
Bettina Kneip \\ Institut für Naturheilkunde, Universitätsspital Zürich, Zürich, Schweiz
}

\section{Schlüsselwörter}

Menopause - Menopausale Symptome · Padma Nerven-Tonikum .

Padma Nervotonin · Traditionelle Tibetische Medizin · TTM · Srog 'zin 10

\section{Zusammenfassung}

Hintergrund: Die Folgen der hormonellen Umstellung in den Wechseljahren der Frau beeinträchtigen die Lebensqualität der Betroffenen erheblich. Viele Frauen wollen oder müssen die Standardtherapie mit Hormonersatzpräparaten vermeiden und bevorzugen komplementäre Behandlungsoptionen. Das pflanzliche Kombinationsarzneimittel Padma Nerven-Tonikum (Srog 'zin 10) wird eingesetzt bei nervösen Zuständen, Reizbarkeit und Ein- und Durchschlafstörungen, d.h. bei Beschwerden, die auch bei peri- und postmenopausalen Frauen vermehrt auftreten. Methoden: Der Behandlungsverlauf wurde anhand des MRS(Menopause Rating Scale)-Fragebogens festgehalten. Es handelt sich dabei um eine Selbstbeurteilungsskala für klimakterische Frauen. Die Daten wurden für Tag 1 der Therapie und nach einem Behandlungszeitraum von 4 Wochen erhoben. Ergebnisse: Im Verlauf der Behandlung von 11 Patientinnen mit menopausalen Beschwerden, mit und ohne onkologischen Vorbefund, zeigte sich durch Padma Nerven-Tonikum - bei guter Verträglichkeit - eine starke Verbesserung verschiedener Symptome, insbesondere von Hitzewallungen, Herzbeschwerden und Schlafstörungen. Ausserdem konnten die Beschwerden Depression, Reizbarkeit und Fatigue reduziert werden. Schlussfolgerungen: Die Resultate zeigen, dass Padma Nerven-Tonikum bei peri- und postmenopausalen Frauen zur Linderung von Wechseljahrbeschwerden eingesetzt werden kann.

\section{Einführung}

Im Lauf der Jahre produzieren die Ovarien immer weniger Östrogene und Progesteron. Als Folge davon treten je nach Prädisposition und individuellen Lebensumständen die Symptome der Wechseljahre mehr oder weniger

\section{Keywords}

Menopause · Menopausal symptoms · Padma Nerves Formula .

Padma Nervotonin · Traditional Tibetan Medicine · TTM · Srog 'zin 10

\section{Summary}

Therapy Evaluation of the Treatment of Menopausal Symptoms with Padma Nerves Formula

Background: The effects of hormonal changes accompanying the menopause in women affect their quality of life significantly. Many women want to or have to avoid standard therapies with hormone replacement preparations and prefer complementary treatment options. The herbal combination product Padma Nerves Formula (Srog 'zin 10) is traditionally used for nervous conditions, irritability, and sleeping disorders, all of which symptoms that are commonly observed in peri- and postmenopausal women. Methods: The course of treatment was assessed using the Menopause Rating Scale (MRS) questionnaire. This questionnaire is a self-rating scale for menopausal women. The data were obtained for day 1 of the treatment and after a treatment period of 4 weeks. Results: In the course of the treatment of 11 patients with menopausal symptoms, with or without initial oncological findings, a significant improvement of symptoms, particularly hot flashes, heart problems, and sleep disorders, could be observed together with a good tolerability of Padma Nerves Formula. Other disorders such as depression, irritability, and fatigue were also reduced. Conclusions: The results indicate that Padma Nerves Formula can be used in peri- and postmenopausal women for the relief of menopausal symptoms.

stark in Erscheinung. Dabei ist der Östrogenabfall für die meisten der menopausalen Symptome verantwortlich, jedoch begünstigen auch Stress und Schlafmangel deren Manifestation. Die Symptome sind meist unangenehm, manchmal gar schmerzvoll und beeinflussen das tägliche Leben der Betroffenen erheblich. Patientinnenbasierte

\section{KARGER}

Fax +497614520714 Information@Karger.com www.karger.com
$1015-0684 / 15 / 0273-0165 \$ 39.50 / 0$
Hofacherstrasse 22, 5443 Niederrohrdorf, Schweiz

info@praxis-kneip.ch 
Studien haben gezeigt, dass Frauen die Wechseljahrbeschwerden als eine starke Beeinträchtigung ihrer Lebensqualität empfinden [1].

Nach wie vor ist die Hormonersatztherapie (engl. «hormone replacement therapy» (HRT)) mit Östrogenpräparaten Standard in der medikamentösen Behandlung von menopausalen Beschwerden [2]. Aufgrund des erhöhten Risikos z.B. für Schlaganfall und venöse Thromoembolie [3] einer solchen Therapie oder weil ein Befund für einen östrogen-sensitiven Brustkrebs vorliegt, wollen oder können viele Frauen nicht mit HRT behandelt werden [4]. Daher besteht ein Bedürfnis nach alternativen, insbesondere pflanzlichen Therapien. In diesem Bereich werden einerseits Präparate mit Phytoöstrogenen wie Soja, Roter Klee oder Traubensilberkerze angewendet, andererseits kommen auch der Symptomatik entsprechende andere Heilpflanzen zum Einsatz, z.B. Hopfen oder Baldrian bei Schlafstörungen oder Weissdorn bei Herz- und Kreislaufbeschwerden.

Padma Nerven-Tonikum ist ein pflanzliches Vielstoffgemisch, das in der Schweiz nach den Traditionen der Tibetischen Medizin hergestellt wird (tibetischer Name Srog 'zin 10). Es ist in der Schweiz im Kanton Appenzell Ausserrhoden als Arzneimittel zugelassen, wirkt nervenstärkend und beruhigend und wird bei Nervosität, Reizbarkeit, innerer Unruhe und Spannungszuständen sowie bei Ein- und Durchschlafstörungen angewendet [5]. Die Rezeptur enthält folgende Wirkstoffe (Mengenangaben pro Kapsel), worunter sich keine bekannten phytoöstrogenhaltigen Pflanzen befinden: $50 \mathrm{mg}$ Asafoetida, $30 \mathrm{mg}$ Gewürznelken, $50 \mathrm{mg}$ Guajakholz, 40 mg Indische Costuswurzel, 30 mg Kaolin, 30 mg Malabarische Wollbaumblüten, $40 \mathrm{mg}$ Muskatnuss, $40 \mathrm{mg}$ entsteinte Myrobalanenfrüchte, $60 \mathrm{mg}$ Nepalesische Mombinpflaumen und $20 \mathrm{mg}$ Weihrauch. In den Sprechstunden des Instituts für Naturheilkunde des Universitätsspitals Zürich wurde das Arzneimittel bei Schlafstörungen eingesetzt, insbesondere auch bei Patientinnen mit Schlafstörungen im Zusammenhang mit menopausalen Beschwerden.

Im Folgenden werden die Resultate aus einer Therapieevaluation der Verlaufsdaten von 11 Patientinnen beschrieben und diskutiert.

Die Beobachtung von peri- und postmenopausalen Frauen mit klimakterischen Beschwerden während der Sprechstunde zeigte, dass sich Symptome der Wechseljahre wie Schlafstörungen, Palpitationen, Reizbarkeit, Hitzewallungen, Depression und Fatigue durch die Einnahme des pflanzlichen Arzneimittels Padma NervenTonikum (Padma Nervotonin) verbesserten. Das Arzneimittel wurde anfänglich gegen Schlafstörungen verschrieben, wobei die beobachtete Verbesserung anderer menopausaler Symptome als positiver Nebeneffekt registriert wurde.

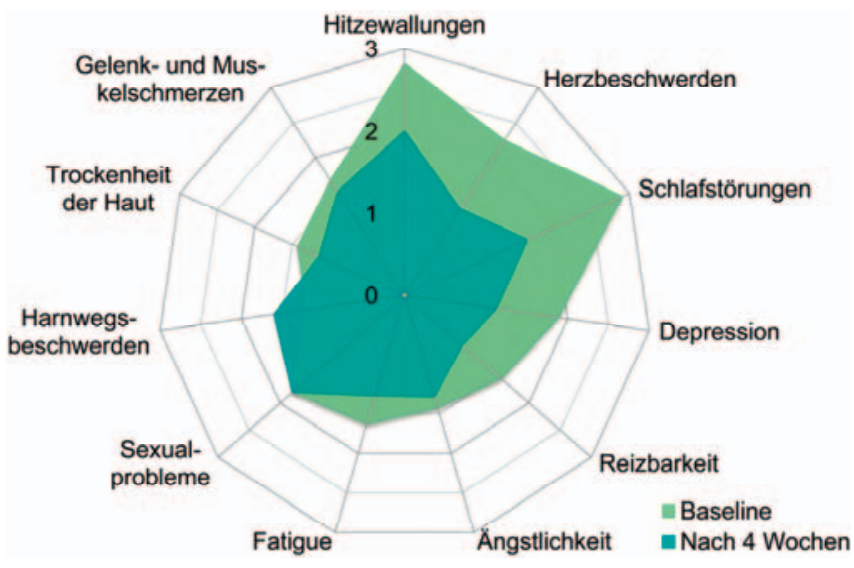

Abb. 1. Verbesserung der Ausprägung menopausaler Symptome nach 4 Wochen Behandlung mit Padma Nerven-Tonikum. Skala: $0=$ keine, $1=$ leichte, 2 = mittlere, 3 = starke Ausprägung der Symptome.

\section{Patienten und Methoden}

Die Therapieevaluation umfasste insgesamt 11 Patientinnen im Alter von 39 bis 66 Jahren (Durchschnitt 53,9 Jahre) mit peri- bzw. postmenopausalen Beschwerden, insbesondere mit Schlafstörungen. Ein Teil der Patientinnen hatte einen onkologischen Vorbefund wie z.B. Mammakarzinom, zum Teil bezüglich Karzinomerkrankung operiert und zum Teil unter Chemo-, Antihormon- und/oder Radiotherapie. Die Dosierung war wie in der Packungsbeilage empfohlen je 2 Kapseln Padma Nerven-Tonikum morgens und abends (Tagesdosis 4 Kapseln).

Zur Überprüfung des Behandlungsverlaufs und -erfolgs wurde der MRS(Menopause Rating Scale)-Fragebogen verwendet. Die Daten wurden für Tag 1 der Therapie und nach einem Behandlungszeitraum von 4 Wochen erhoben.

Beim MRS-Fragebogen handelt es sich um eine Selbstbeurteilungsskala für klimakterische Frauen. Er ist ein effizientes Mittel für die Diagnostik und Quantifizierung von Wechseljahrbeschwerden und beinhaltet die individuelle Bewertung von Symptomen auf einer Skala von 0 bis 4 (keine / leichte / mittlere / starke / sehr starke Beschwerden) [6].

Während der Behandlung mit Padma Nerven-Tonikum fand keine Co-Medikation mit anderen pflanzlichen Arzneimitteln oder Hormonen gegen Wechseljahrbeschwerden statt.

\section{Resultate}

Die am stärksten ausgeprägten und häufigsten Beschwerden in dieser Patientinnengruppe waren Schlafstörungen, Hitzewallungen, Herzbeschwerden und Depression. Diese Symptome zeigten über den Behandlungszeitraum eine starke Verbesserung mit einer Reduktion der durchschnittlichen Intensität um jeweils 1,27, 0,82, 1 und 0,77 Skalenpunkte (Abb. 1). Auch die Ausprägung des Symptoms Reizbarkeit reduzierte sich merklich um 0,63 Punkte, und in einem geringeren Mass verbesserten sich Trockenheit der Haut und der Schleimhaut, Fatigue, Ängstlichkeit sowie Gelenk- und Muskelschmerzen (jeweils um 0,29, 0,36, 0,14 und 0,17 Punkte). 


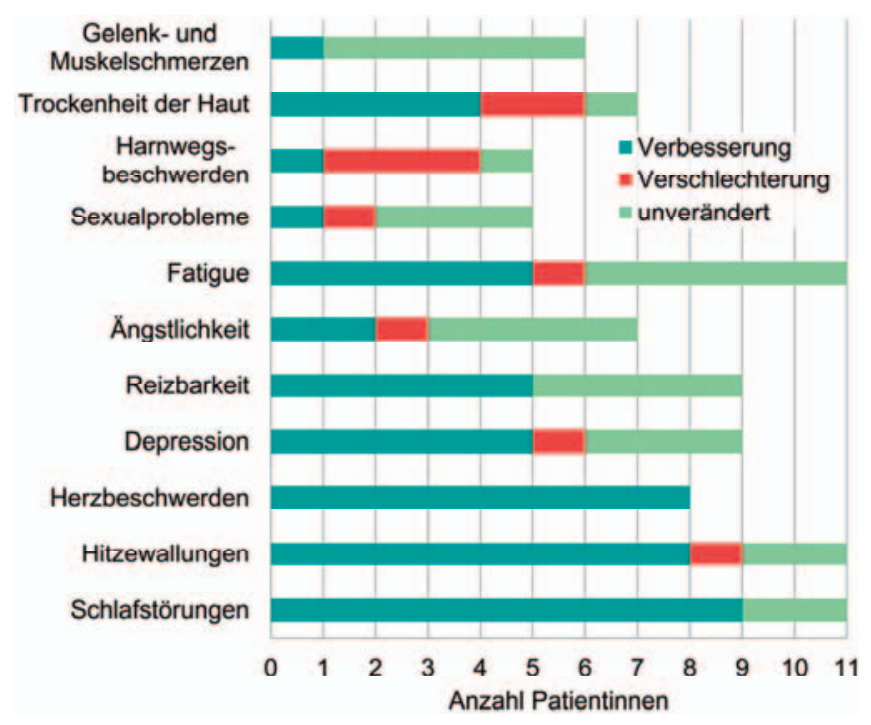

Abb. 2. Anzahl der Patientinnen, welche beim entsprechenden Symptom eine Verbesserung, eine Verschlechterung oder keine Veränderung feststellten.

Sexualprobleme und Harnwegsbeschwerden traten jeweils bei 5 der 11 Patientinnen auf, wobei sich die Ausprägung dieser Sexualprobleme während der Therapie nicht veränderte. Dagegen zeigte sich beim Symptom Harnwegsprobleme bei 3 Patientinnen eine Verschlechterung, bei einer Patientin eine Verbesserung und bei einer keine Veränderung der Ausprägung. Die Verbesserung zeigte sich bei der einzigen dieser Patientinnen, die das Symptom vor der Therapie in starker Intensität hatte (Reduktion von stark auf mittel).

Die Mehrheit der Patientinnen konnte von einer Verbesserung der Symptome Hitzewallungen, Herzbeschwerden und Schlafstörungen profitieren (Abb. 2), während bei Depression, Reizbarkeit und Fatigue etwa die Hälfte eine Verbesserung und die andere Hälfte keine Veränderung feststellen konnte. Die übrigen untersuchten Symptome blieben bei den meisten Patientinnen unverändert. Es wurden keine Nebenwirkungen berichtet.

\section{Schlussfolgerung}

Die durch eine Abnahme des Östrogen- und Progesteronspiegels im Blut auftretenden Wechseljahrbeschwerden werden von den betroffenen Patientinnen meist als unangenehm, manchmal sogar als schmerzhaft empfunden und beeinträchtigen deren Lebensqualität erheblich. Die vorliegende Studie zeigt, dass das Tibetische Arzneimittel Padma Nerven-Tonikum eine deutliche Verbesserung verschiedener menopausaler Beschwerden bewirkte, insbesondere Schlafstörungen, Hitzewallungen und Herzbeschwerden, und auch die Symptome Reizbarkeit, Depression und Fatigue günstig beeinflusste - sowohl bei Patientinnen mit als auch bei solchen ohne onkologische Vorgeschichte. Die Verträglichkeit war gut. Eine Bestätigung dieser Resultate im Rahmen einer klinischen Studie wäre wünschenswert. Trotz der relativ geringen Fallzahl zeigen die Ergebnisse, dass Padma Nerven-Tonikum eine Option in der Behandlung von Wechseljahrbeschwerden bei peri- und postmenopausalen Frauen darstellt, insbesondere wenn eine Alternative zur HRT gewünscht oder notwendig ist.

\section{Disclosure Statement}

Die Studie wurde im Zusammenhang mit der Abschlussarbeit für die Schweizerische Medizinische Gesellschaft für Phytotherapie (SMGP) durchgeführt. Für die Autorin bestehen keinerlei Interessenkonflikte.

\section{Literatur}

1 Blumel JE, Castelo-Branco C, Binfa L, Gramegna G, Tacla X, Aracena B, Cumsille MA, Sanjuan A: Quality of life after the menopause: a population study. Maturitas 2000; 34:17-23.

2 Arbeitsgemeinschaft der Wissenschaftlichen Medizinischen Fachgesellschaften: S3-Leitlinie Hormontherapie (HT) in der Periund Postmenopause. AWMF 015/062. www. awmf.org/leitlinien/detail/ll/015-062.html.
Manson JE: Current recommendations: what is the clinician to do? Fertil Steril 2014;101: 916-921.

4 Pitkin J: Alternative and complementary therapies for the menopause. Menopause Int 2012;18:20-27.
5 Packungsbeilage Padma Nerven-Tonikum. www.padma.ch/produkte/weitere-produkte-ch/ kantonale-arzneimittel/padma-nerven-tonikum.

6 Heinemann L, Potthoff P, Schneider H: International versions of the Menopause Rating Scale (MRS). Health Qual Life Outcomes 2003; $1: 28$. 